\title{
Decision-making in pulmonary endarterectomy surgery
}

\author{
To the Editor:
}

We would like to thank N.H. Kim and E. Mayer for their editorial based on our recent paper "The impact of patient choice on survival in chronic thromboembolic pulmonary hypertension" [1,2]. We agree with many of their comments pertaining to the importance of referring patients diagnosed with chronic thromboembolic pulmonary hypertension (CTEPH) for assessment by a pulmonary endarterectomy (PEA) programme. We would, however, like to correct certain factual errors in their editorial which we feel create an incorrect picture of our practice at a large tertiary pulmonary hypertension referral centre.

First, N.H. Kim and E. Mayer state that following remote assessment by a surgeon at our national PEA centre, $278(50 \%)$ out of 550 of patients were deemed not to be candidates for PEA surgery. In fact, figure 1 in our original article [1] shows that $81 \%$ (448 out of 550) of patients were deemed by our national CTEPH multidisciplinary team (MDT) to have potentially surgical disease, while 19\% (102 out of 550) were felt to have inoperable disease due to disease distribution. This figure was very similar to the $21 \%$ (140 out of 679) of the international CTEPH registry patients who were not offered surgery because of disease distribution when compared to pulmonary haemodynamic derangement [3]. Overall, the proportion of patients in our study who did not undergo PEA for reasons other than disease distribution and patient choice was not dissimilar to that observed in the international CTEPH registry (17\% versus $12 \%)$.

Second, N.H. Kim and E. Mayer are mistaken in stating that the 72 patients who declined surgery did so before meeting the surgical team. Nowhere in our manuscript is this statement supported and we would like to clarify our referral pathway. Within the UK, patients are diagnosed with CTEPH at one of seven pulmonary vascular disease units following rigorous assessment involving multi-modality imaging and right heart catheterisation. Our approach is then to send clinical details, haemodynamic data and radiology for all patients diagnosed with CTEPH (including those who their pulmonary vascular disease unit think may have inoperable disease) to the CTEPH MDT meeting at Papworth, the national PEA centre. This MDT involves pulmonary vascular radiologists, physicians and nurses together with PEA surgeons. Patients who are deemed to have potentially operable disease are invited to meet the surgical team at Papworth. A proportion of patients are deemed to have inoperable disease (be it due to disease distribution or comorbidities) following discussion at the surgical MDT, although where there is clinical doubt these patients too will be offered review at Papworth. Only after a face-to-face surgical review are patients asked to make a decision regarding surgery. This referral pathway, via nationally designated pulmonary vascular disease units to a single national CTEPH MDT, maximises the likelihood that a patient in the UK will be offered PEA. Indeed, this model of care has resulted in PEA rates that, based on numbers of PEA procedures performed annually compared with the national population (2.7 procedures per million population in 2017), may be the highest in the world.

Finally, N.H. Kim and E. Mayer state in their final paragraph that those specialists who make frequent initial diagnoses of CTEPH need ongoing education and better understanding of CTEPH treatment. We agree that the international physician survey of CTEPH management they reference does present concerning data regarding low levels of referral for PEA surgery [4]. It must be noted, however, that the physician cohort in that study was comprised of doctors previously registered with a market research company who had to self-declare only low levels of CTEPH diagnosis and management, rather than a high-volume, nationally designated pulmonary hypertension centre as in our study. Nevertheless, we would strongly agree that a physician making a diagnosis of CTEPH is duty bound to fully counsel their

@ERSpublications

Clarification of certain issues related to the UK pulmonary endarterectomy referral pathway, emphasising the importance of referral of all patients with CTEPH to a surgical team http://ow.ly/ Db6I30mwbVP

Cite this article as: Condliffe R, Quadery SR, Jenkins DP, et al. Decision-making in pulmonary endarterectomy surgery. Eur Respir J 2019; 53: 1801973 [https://doi.org/10.1183/13993003.01973-2018]. 
patient about the optimal management of CTEPH and the importance of a timely referral to an expert PEA centre. We agree that an opportunity to meet with the surgical team is a key part of this process, but this study highlights that despite this, some patients do choose not to proceed down the route of surgery. Further work is required to understand factors influencing decision-making including how information is provided to patients, how individuals perceive risk and how to ensure that the individual makes a genuinely informed decision. We hope that the data presented in our study demonstrating the clear survival benefit in choosing surgery if offered will be of use to physicians and patients during this decision-making.

Robin Condliffe ${ }^{1}$, Syed Rehan Quadery ${ }^{1}$, David P. Jenkins ${ }^{2}$, Iain J. Armstrong ${ }^{1,3}$ and David G. Kiely ${ }^{1}$

${ }^{1}$ Sheffield Pulmonary Vascular Disease Unit, Royal Hallamshire Hospital, Sheffield Teaching Hospitals NHS Foundation Trust, Sheffield, UK. ${ }^{2}$ Pulmonary Vascular Disease Unit, Papworth Hospital NHS Foundation Trust, Cambridge, UK.

${ }^{3}$ Pulmonary Hypertension Association, UK.

Correspondence: Robin Condliffe, Sheffield Pulmonary Vascular Disease Unit, Royal Hallamshire Hospital, Sheffield, S10 2JF, UK. E-mail: robin.condliffe@sth.nhs.uk

Received: Oct 172018 | Accepted: Oct 212018

Conflict of interest: R. Condliffe reports personal fees and non-financial support (advisory boards and honoraria for lecturing) from Actelion, GSK and MSD, and non-financial support (honoraria for lecturing) from Bayer, outside the submitted work. S.R. Quadery has nothing to disclose. D.P. Jenkins reports personal fees from Actelion (lecturing and consulting fees) and personal fees from Bayer (lecturing fees), outside the submitted work. I.J. Armstrong has nothing to disclose. D.G. Kiely reports departmental grants, personal fees (lecturing and consulting fees) and non-financial support from Actelion, GSK, MSD and Bayer, outside the submitted work.

\section{References}

1 Quadery SR, Swift AJ, Billings CG, et al. The impact of patient choice on survival in chronic thromboembolic pulmonary hypertension. Eur Respir J 2018; 52: 1800589.

2 Kim NH, Mayer E. Pulmonary endarterectomy and the cost of patient refusal. Eur Respir J 2018; 52: 1801581.

3 Delcroix M, Lang I, Pepke-Zaba J, et al. Long-term outcome of patients with chronic thromboembolic pulmonary hypertension: results from an international prospective registry. Circulation 2016; 133: 859-871.

4 Gall H, Preston IR, Hinzmann B, et al. An international physician survey of chronic thromboembolic pulmonary hypertension management. Pulm Circ 2016; 6: 472-482.

From the authors:

We thank R. Condliffe and co-workers for their critical clarifications and for pointing out any potential misrepresentation of their report [1]. We recognised in our review of the manuscript that $81 \%$ upon remote review were felt to be technically operable cases. However, in the end, only half were operated. In their response, R. Condliffe and co-workers provide further elaboration of their chronic thromboembolic pulmonary hypertension (CTEPH) case review process, in close partnership with the surgical centre at Papworth, including the important offer of an in-person consultation. So, were all 135 patients (72 who refused and 63 who were deemed unfit for surgery) examined and reviewed in person at the surgical reference centre? This is critical information left out in the original paper and in their correspondence, and the point which should be highlighted in this dialogue.

CTEPH operability assessment remains highly subjective and especially difficult to do remotely. These were key considerations why all major trials of medical therapy for inoperable CTEPH required an adjudication committee of experts [2-4]. However, even among experts there can be disagreements [5]. The adjudication committee from the CHEST- 1 study noted that $22 \%$ of patients ( 69 of 312 cases) who were initially deemed inoperable by experts, either the central or designated adjudication committees,

@ERSpublications

Operability assessment in CTEPH remains highly subjective. In order to provide the optimal care to each patient with CTEPH, we encourage direct evaluation and counselling whenever possible. http://ow.ly/uRCO30mMs46

Cite this article as: Kim NH, Mayer E. Decision-making in pulmonary endarterectomy surgery. Eur Respir J 2019; 53: 1802138 [https://doi.org/10.1183/13993003.02138-2018]. 
upon second review were changed to operable. So even in optimal circumstances with known experts, operability assessment is not infallible. In recognition, multiple CTEPH treatment algorithms have incorporated a recommendation to obtain a second opinion $[6,7]$. In practice, we routinely witness cases deemed inoperable elsewhere or uncertain upon remote review that undergo successful pulmonary endarterectomy following local re-examination of the patient. Therefore, we commend the authors and their programmes for having the face-to-face consultation built into their routine practice.

However, if not all 135 patients were seen at Papworth before making an ultimate decision regarding surgery, it would help to know the details, and we encourage the authors to look at that data more carefully. If majority of the refusals declined an in-person evaluation at the reference centre, that data would be informative and potentially practice altering. If, on the other hand, many visited the reference centre and still refused surgery, what was the breakdown of refusal between the group that visited Papworth for evaluation and those who declined the visit? Is it possible that patients who refuse medical recommendations, such as visiting the reference centre to determine candidacy, are inherently higher risk long-term? But if all, or nearly all, of the 135 in fact were seen directly at the reference centre, that information may be of value to Papworth in their pursuit of treating as many technically operable cases as possible, as compelled by the data on the differences in survival. Finally, in recognition of the growing complexity of CTEPH treatment with expansion of numerous nonsurgical treatment modalities, along with the emphasis on the importance of pulmonary endarterectomy as spearheaded in this report, we recommend a message to all medical providers to refer CTEPH cases for comprehensive treatment evaluations at expert treatment centres, which are not only capable of investigating the nuances of each CTEPH case, but counsel patients effectively drawing from vast experience on the most appropriate therapy for that individual patient.

Nick H. Kim ${ }^{1}$ and Eckhard Mayer ${ }^{2}$

${ }^{1}$ Pulmonary and Critical Care Medicine, University of California San Diego, La Jolla, CA, USA. ${ }^{2}$ Thoracic Surgery, Kerckhoff Clinic Heart and Lung Centre, Bad Nauheim, Germany.

Correspondence: Nick H. Kim, Pulmonary and Critical Care Medicine, University of California San Diego, 9300 Campus Point Drive, MC 7381, La Jolla, CA 92037, USA. E-mail: h33kim@ucsd.edu

Received: Nov 082018 | Accepted: Nov 082018

Conflict of interest: None declared.

\section{References}

1 Quadery SR, Swift AJ, Billings CG, et al. The impact of patient choice on survival in chronic thromboembolic pulmonary hypertension. Eur Respir J 2018; 52: 1800589.

2 Jais X, D'Armini AM, Jansa P, et al. Bosentan for treatment of inoperable chronic thromboembolic pulmonary hypertension: BENEFiT (Bosentan Effects in iNopErable Forms of chronIc Thromboembolic pulmonary hypertension), a randomized, placebo-controlled trial. J Am Coll Cardiol 2008; 52: 2127-2134.

3 Ghofrani HA, D'Armini AM, Grimminger F, et al. Riociguat for the treatment of chronic thromboembolic pulmonary hypertension. N Engl J Med 2013; 369: 319-329.

4 Ghofrani HA, Simonneau G, D'Armini AM, et al. Macitentan for the treatment of inoperable chronic thromboembolic pulmonary hypertension (MERIT-1): results from the multicentre, phase 2, randomised, double-blind, placebo-controlled study. Lancet Respir Med 2017; 5: 785-794.

5 Jenkins DP, Biederman A, D'Armini AM, et al. Operability assessment in CTEPH: lessons from the CHEST-1 study. J Thorac Cardiovasc Surg 2016; 152: 669-674.

6 Kim NH, Delcroix M, Jenkins DP, et al. Chronic thromboembolic pulmonary hypertension. J Am Coll Cardiol 2013; 62: Suppl. 25, D92-D99.

7 Wilkens H, Konstantinides S, Lang IM, et al. Chronic thromboembolic pulmonary hypertension (CTEPH): updated recommendations from the Cologne Consensus Conference 2018. Int J Cardiolol 2018; in press [https://doi.org/10. 1016/j.ijcard.2018.08.079]. 